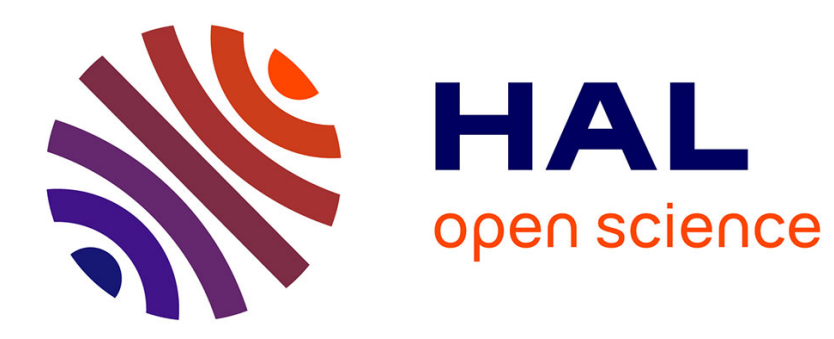

\title{
Le gouvernement fédéral américain au chevet de l'assurance santé
}

\author{
Anne-Laure Beaussier
}

\section{To cite this version:}

Anne-Laure Beaussier. Le gouvernement fédéral américain au chevet de l'assurance santé. La Revue de l'IRES, 2021, № 103-104 (1), pp.109-129. 10.3917/rdli.103.0109 . hal-03514045

\section{HAL Id: hal-03514045 \\ https://hal.science/hal-03514045}

Submitted on 19 Jan 2022

HAL is a multi-disciplinary open access archive for the deposit and dissemination of scientific research documents, whether they are published or not. The documents may come from teaching and research institutions in France or abroad, or from public or private research centers.
L'archive ouverte pluridisciplinaire HAL, est destinée au dépôt et à la diffusion de documents scientifiques de niveau recherche, publiés ou non, émanant des établissements d'enseignement et de recherche français ou étrangers, des laboratoires publics ou privés. 


\title{
LE GOUVERNEMENT FÉDÉRAL AMÉRICAIN AU CHEVET DE L'ASSURANCE SANTÉ
}

\author{
Anne-Laure Beaussier
}

\author{
I.R.E.S. | « La Revue de l'Ires »
}

2021/1 N¹03-104 | pages 109 à 129

ISSN 1145-1378

DOI 10.3917/rdli.103.0109

Article disponible en ligne à l'adresse :

https://www.cairn.info/revue-de-l-ires-2021-1-page-109.htm

Distribution électronique Cairn.info pour I.R.E.S..

(C) I.R.E.S.. Tous droits réservés pour tous pays.

La reproduction ou représentation de cet article, notamment par photocopie, n'est autorisée que dans les limites des conditions générales d'utilisation du site ou, le cas échéant, des conditions générales de la licence souscrite par votre établissement. Toute autre reproduction ou représentation, en tout ou partie, sous quelque forme et de quelque manière que ce soit, est interdite sauf accord préalable et écrit de l'éditeur, en dehors des cas prévus par la législation en vigueur en France. Il est précisé que son stockage dans une base de données est également interdit. 


\title{
Le gouvernement fédéral américain au chevet de l'assurance santé
}

\author{
Anne-Laure BEAUSSIER ${ }^{1}$
}

Pour beaucoup d'observateurs des politiques de santé des États-Unis, les 28 millions de personnes restant privées d'assurance maladie en 2020, dix ans après l'adoption de l'Obamacare, la réforme du système de santé emblématique du premier mandat de Barack Obama, constituent une démonstration d'échec, appelant à une réforme de la réforme. L'Affordable Care Act (ACA) avait pourtant fait le pari qu'il était possible, avec les bons instruments réglementaires et incitatifs, non seulement de soutenir et de reconstruire un marché des assurances santé qui semblait au bord de l'explosion mais également de l'inciter à endosser des objectifs de service public et de bien commun. Dix ans plus tard cependant, les limites de la réforme, à la fois en termes de couverture de la population et en termes de maîtrise des dépenses de santé alimentent de nombreuses critiques. Cette contribution revient sur la stratégie suivie par l'ACA. Elle explicite ses principaux instruments, analyse les difficultés rencontrées lors de leur mise en œuvre et discute les alternatives envisagées aujourd'hui tant du côté démocrate que du côté républicain.

Lorsque l'on pense au système d'assurance santé américain, on a surtout à l'esprit un système privé dans lequel rôle de l'État est minimal, intervenant au mieux ponctuellement lorsque le secteur privé n'est pas en mesure de prendre en charge le risque maladie de certains groupes de population. Si les assurances privées constituent la forme dominante de protection maladie ${ }^{2}$, l'État américain y occupe néanmoins, et de plus en plus, un rôle de premier plan. Ce rôle est double : il tient d'abord à la prise en charge

1. Chargée de recherche au CNRS, Centre de sociologie des organisations, Sciences Po Paris. Je souhaite remercier chaleureusement Catherine Sauviat pour ses commentaires et suggestions de modifications qui ont considérablement amélioré cet article et ma perspective sur ce sujet.

2. Elles représentent aujourd'hui encore $55 \%$ de couverture de la population, soit environ 177 millions de personnes. 
directe de la population au travers de programmes fédéraux dont les plus importants sont Medicare et Medicaid ${ }^{3}$ - couvrant aujourd'hui environ 122 millions de personnes, soit un Américain sur trois (Congressional Research Service, 2021) ${ }^{4}$. Ensuite, plus indirectement, le gouvernement fournit un soutien à la fois financier et réglementaire au marché des assurances santé afin d'étendre et d'améliorer la couverture maladie proposée par le secteur privé et améliorer les conditions de la compétition entre opérateurs privés. Pour reprendre une expression de Howard (1997), ce dernier volet, qui est monté en puissance au cours des dernières décennies, s'inscrit dans une logique d'« État-providence invisible».

Les premières politiques ciblées sur le marché des assurances privées remontent aux années 1950 sous la présidence d'Eisenhower. L'administration républicaine initie alors une politique d'exonérations fiscales à destination des employeurs proposant des assurances santé collectives à leurs salariés et à destination des salariés eux-mêmes qui peuvent déduire de leur déclaration de revenus leur contribution à leur police d'assurance professionnelle. Visant à encourager la croissance des assurances privées, jusque-là peu répandues, ces mesures fiscales, renforcées en 1974 sous la présidence de Nixon par la loi Employee Retirement Income Security Act (ERISA), ont un impact considérable sur le développement des assurances santé, en particulier le segment des assurances collectives professionnelles. Alors que seulement $10 \%$ de la population a une assurance santé dans les années 1950, cette proportion atteint $80 \%$ dans les années 1970 et les assurances collectives professionnelles constituent un peu plus de $90 \% \mathrm{de}$ ce total.

Les actions de soutien et d'encadrement du marché des assurances deviennent progressivement une dimension essentielle de l'action publique dans le secteur de la santé. D'autant plus essentielle qu'à partir des années 1980 et surtout 1990, le marché des assurances santé commence à montrer des signes d'essoufflement. Une conjoncture économique plus difficile couplée à une explosion des dépenses de santé ${ }^{5}$ entraîne un repli des assurances santé en particulier collectives proposées par les sociétés américaines ${ }^{6}$. Entre 1980 et 1995, le prix moyen d'une assurance santé augmente de plus du double. Les employeurs sont moins nombreux à

3. Medicare assure l'ensemble des Étasuniens de plus de 65 ans - soit en $2019,18 \%$ de la population (58 millions de personnes ; Congressional Research Service, 2021), sur la base de cotisations sociales versées au cours de la vie professionnelle. Medicaid est une couverture santé ciblée sur les plus pauvres, financée par l'impôt au niveau fédéral et gérée concrètement par chacun des 50 États fédérés. En 2018, cette politique couvrait près de 65 millions de personnes, soit $20 \%$ de la population des États-Unis.

4. II faut ajouter à ces programmes 4,5 millions de vétérans des armées couverts par les assurances maladies de la Veteran Administration.

5. Celles-ci passent de $4 \%$ dans les années 1950 à $17 \%$ du PIB en 2009, avec des taux de croissance annuels entre 4 et $5 \%$.

6. Le taux de couverture de la population des assurances professionnelles chute, passant de $72 \%$ de la population en 1980 à $49 \%$ en 2018 . 
proposer des assurances santé à leurs salariés, surtout chez les petites et moyennes entreprises pour qui ces bénéfices constituent une charge financière importante. Plus chères et moins répandues que les assurances collectives, les assurances individuelles ne parviennent pas à compenser le repli des assurances santé professionnelles et les programmes publics, tels que Medicaid, connaissent une croissance importante sans pour autant absorber le flot d'individus ayant perdu leur accès aux soins via leur employeur. Ces années voient ainsi la proportion de personnes sans assurance santé augmenter à un rythme soutenu de $3 \%$ par an. Au début des années 2010, au moment où l'Obamacare est votée, près de 50 millions de personnes n'ont plus de protection maladie, auxquels s'ajoutent 20 millions de personnes «mal assurées ».

L'Affordable Care Act de 2010, aussi appelée Obamacare, cherche à transformer en profondeur le secteur des assurances santé pour en corriger les dysfonctionnements les plus importants, les rendre plus abordables, plus disponibles et de meilleure qualité pour les Américains et ainsi pour mener le pays sur la voie d'une couverture maladie universelle. En plus d'étendre Medicaid à un nombre croissant d'Américains aux revenus modestes, la réforme renforce les subventions et exonérations fiscales destinées aux personnes rencontrant le plus de difficultés pour s'assurer : les travailleurs indépendants, les petites et moyennes entreprises ainsi que les individus ne bénéficiant pas d'une assurance santé liée à leur emploi et contraints de s'assurer individuellement. Selon les calculs du Congressional Budget Office (CBO, 2019), l'ensemble des exonérations fiscales liées aux assurances collectives et à l'Obamacare représentent en 2019 un total de 350 milliards de dollars (soit 289 milliards d'euros; respectivement 287 milliards de dollars, ou 238 milliards d'euros, pour l'ERISA et 62 milliards de dollars, soit 51 milliards d'euros, pour l'Obamacare), faisant de ces actions le troisième poste de dépenses du gouvernement fédéral en matière de santé après Medicare et Medicaid. Au-delà d'incitations financières ciblées sur la demande d'assurances, l'Obamacare cherche à assigner au marché des assurances des objectifs de solidarité ou d'intérêt général en réglementant l'offre d'assurance : encadrant les pratiques des assureurs privés, créant des standards de qualité minimaux, restreignant la possibilité d'une tarification au risque et prohibant les pratiques de discrimination sur la base d'antécédents médicaux.

Ces ambitions se sont-elles réalisées aujourd'hui ? Cette conjonction entre subventions et réglementation a-t-elle permis aux Américains privés d'accès aux soins de bénéficier d'une protection maladie accessible et de qualité ? Dix ans après sa mise en place, aux termes du mandat de Donald Trump, chaotique et ouvertement hostile à l'Obamacare, quel a été l'impact de la réforme sur les assurances privées? Comme nous le montrons 
plus bas, ce bilan est en demi-teinte. Si des gains de couverture importants ont été obtenus, une assurance universelle de la population est toujours un horizon lointain. En dépit des régulations et subventions, le prix des polices d'assurance continue d'augmenter à un rythme soutenu et la qualité des couvertures proposées demeure hétérogène. La suite de cet article détaille les mécanismes de soutien au marché introduits par l'Obamacare et leur place dans l'architecture plus générale de la réforme. Il en montre ses avancées et limites, avant de discuter les propositions soutenues par le nouveau Président démocrate Joe Biden pour prolonger la réforme et en corriger les faiblesses.

\section{L'ambition d'Obamacare : un meilleur accès aux soins et des objectifs de santé publique}

Comme évoqué ci-dessus, l'Obamacare a été plus loin par sa portée et par son ambition qu'aucune autre réforme avant elle dans la régulation, l'encadrement et le soutien au secteur des assurances maladies et à l'assurance privée. Ce n'est pas la première proposition pour réformer le système d'assurance santé du pays et si on la compare à d'autres tentatives comme la réforme Clinton de 1993-1994, ce n'est pas non plus la plus ambitieuse. Elle puise ainsi certaines de ses idées les plus structurantes dans un répertoire de réformes conservateur. Par exemple, le cœur de l'Obamacare, une obligation individuelle d'assurance faisant peser sur chaque individu la responsabilité de souscrire une assurance santé ou d'être financièrement pénalisé, trouve ses origines dans un think tank américain appartenant à la droite conservatrice, la Heritage Foundation, qui formule le premier cette proposition dans les années 1990 (Butler, Haislmaier, 1989) comme alternative aux propositions démocrates plus ambitieuses visant à renforcer la responsabilité collective des entreprises en créant une obligation pour les employeurs d'assurer leurs salariés. L'Obamacare adopte une approche plus consensuelle et de fait, de toutes les propositions pour créer une assurance santé universelle qui voient le jour dans les années 1990 et 2000, elle est la seule qui ait su rassembler suffisamment de soutien politique et de soutien dans le monde économique pour être votée (Beaussier, 2016) ${ }^{7}$.

Cette réforme est très complexe et ses dispositions impactent la plupart des aspects du système de santé étasunien, qu'il s'agisse de l'organisation de l'offre de soins, du contrôle de la qualité et des dépenses de santé, des soins de prévention, de fin de vie, bien sûr de l'accès aux soins. Il serait trop

7. Avant elle, Bill Clinton tente sans succès en 1993-1994 d'initier une réforme ambitieuse qui aurait obligé tous les employeurs à fournir une assurance santé à leurs salariés, rapprochant le système de santé américain d'un système plus unifié et fondé sur l'emploi et les contributions des employeurs et salariés. Cette obligation est censée être couplée à des subventions supplémentaires notamment pour les petites et moyennes entreprises et à des mesures de contrôle et de limitation des prix des polices d'assurance et des tarifs des professionnels de santé. 
long ici d'en synthétiser l'ensemble des mesures, nous nous contenterons de rappeler les principales mesures sur ce dernier aspect - améliorer l'accès aux soins de la population et parvenir à une couverture presque universelle de la population. Ce volet repose sur trois piliers principaux qui ensemble doivent améliorer l'accès de la population à l'assurance maladie, limiter la croissance des dépenses de santé et améliorer la qualité des soins médicaux proposés.

Le premier pilier est un pilier public. Il élargit assez considérablement l'accès au programme Medicaid aux foyers à revenus modestes (jusqu'à $138 \%$ du seuil de pauvreté fédéral $\left.{ }^{8}\right)$ et harmonise les critères d'éligibilité et garanties de couverture entre les États ayant choisi de participer au dispositif. Ce programme, géré par les États fédérés, était jusque-là très hétérogène selon les territoires et si des États comme New York ou le Massachussetts proposaient une couverture relativement ouverte et généreuse aux personnes à faibles revenus, un grand nombre d'États avait mis en œuvre des conditions d'éligibilité très restrictives, excluant de fait les personnes seules et sans enfant quel que soit leur niveau de revenu.

Le deuxième pilier concerne la demande de soins et crée une double obligation d'assurance qui vise à faire en sorte que le plus grand nombre ait accès et souscrive effectivement une assurance santé. Il s'agit tout d'abord, comme évoqué précédemment, d'une obligation pour l'ensemble des Américains de bénéficier d'une assurance santé sous une forme ou sous une autre (publique, privée, collective ou individuelle) répondant à des critères de qualité minima et couvrant un ensemble de soins jugés essentiels 9 . La seconde, moins connue, est une obligation pour les entreprises de plus de 50 salariés de proposer à l'ensemble de leurs salariés à temps plein une assurance devant également répondre à des standards minima de qualité et d'accessibilité ${ }^{10}$. Dans les deux cas, le non-respect de ces obligations entraîne des pénalités financières (de l'ordre de 2000 dollars, soit 1652 euros, par salarié pour une entreprise et de 700 dollars, soit 578 euros, par adulte

8. Soit un peu plus de 17000 dollars annuels (14043 euros) pour une personne seule. Cela représente un peu moins de $50 \%$ du revenu médian fédéral qui est de 36000 dollars par an pour un individu (29894 euros). Pour plus d'informations, voir : https://www.census.gov/library/publications/ 2020/demo/p60-270.html.

9. Ces assurances doivent couvrir un panier de soins minimal, appelé " essential health benefits » et incluant obligatoirement une couverture hospitalière, une couverture des soins médicaux de ville, de services pédiatriques et obstétriques, de médecine préventive, de soins de psychiatrie, de remboursement des médicaments et d'analyses médicales, de soins d'urgence. Elles doivent en outre répondre à des critères d'accessibilité, fixant des limites dans les frais non pris en charge et frais administratifs facturés par les assureurs. Seuls les individus de moins de 30 ans sont exemptés de ce type d'assurance et peuvent souscrire des contrats ne couvrant que les frais très élevés (" catastrophic care ").

10. Ces standards minima sont identiques à ceux pour les individus. Les entreprises toutefois ont également l'obligation de proposer des assurances abordables économiquement à leurs salariés, c'est-à-dire que la part restant à charge du salarié ne doit pas dépasser $9,8 \%$ de ses revenus annuels. Toutefois, certains contrats existant avant l'entrée en vigueur de l'Obamacare ( "grandfather plans ») et ne respectant pas l'ensemble de ces exigences sont autorisés par la loi. Pour plus de détails voir : https://www.kff.org/infographic/employer-responsibility-under-theaffordable-care-act/. 
dans le cas de l'obligation individuelle d'assurance ${ }^{11}$ ). En contrepartie de ces obligations, l'Obamacare crée un régime de subventions et d'exemptions fiscales généreux s'ajoutant au régime d'exemptions existant sous la loi ERISA de 1974. Ces subventions et crédits d'impôts s'adressent aux individus souhaitant s'assurer sur le segment des assurances individuelles et dont les revenus se situent entre 130 et $400 \%$ du seuil de pauvreté fédéral. En outre, les travailleurs indépendants et les petites et moyennes entreprises de moins de 25 salariés exemptés de l'obligation d'assurance bénéficient également de réductions fiscales s'ils souhaitent proposer une assurance à leurs salariés, pouvant atteindre $50 \%$ du coût de la contribution de l'employeur afin de les inciter à offrir une couverture santé à leurs salariés.

Le troisième pilier enfin est dirigé sur l'offre d'assurance. Il réglemente et impose des standards de qualité et d'accessibilité aux assurances santé, limitant les pratiques d'évitement du risque, de tarification en fonction de l'état de santé des individus, et impose des limites sur les montants engagés dans le remboursement des soins de leurs assurés. Ces réglementations couvrent à la fois les assurances souscrites par les entreprises de plus de 50 salariés et celles souscrites par les individus et les PME de moins de 25 salariés percevant les subventions et exemptions fiscales prévues par l'Obamacare. Ces derniers sont en outre encouragés à souscrire leur assurance au sein de centrales d'achats mettant en compétition différentes assurances certifiées par les pouvoirs publics, les Health Insurance Marketplaces du côté des individus et les Small Business Health Options Program Marketplaces (SHOP Exchanges) pour les travailleurs indépendants et les PME. Les assurances proposées au sein de ces marketplaces sont encadrées par les pouvoirs publics. Elles répondent à des garanties minimales de couverture et ne peuvent refuser de couvrir les personnes présentant des antécédents médicaux ou leur proposer des tarifs prohibitifs (community rating).

Ensemble, ces trois piliers mêlant des instruments de nature réglementaire et des incitations financières et économiques ont permis d'améliorer considérablement l'accès aux soins et le taux de personnes bénéficiant d'une assurance santé (Glied et al., 2020).

Dix ans après l'adoption de l'Obamacare, 20 millions de personnes ont gagné une assurance santé (Tolbert et al., 2020) en dépit des nombreuses tentatives républicaines et de l'administration Trump d'abroger la réforme. Dans le détail, ces gains de couverture sont d'abord liés à l'expansion de Medicaid et, dans une moindre mesure, aux dispositifs de soutien et de

11. Comme nous l'expliquons dans la suite de cet article, la majorité républicaine a supprimé en 2017 la pénalité pesant sur les individus en cas de non-respect de l'obligation individuelle d'assurance. En revanche la pénalité pesant sur les entreprises de plus de 50 salariés subsiste. 


\section{Tableau 1. Principales mesures de l'Obamacare encadrant et facilitant l'accès aux assurances santé *}

\begin{tabular}{|c|c|}
\hline $\begin{array}{l}\text { Type } \\
\text { de mesures }\end{array}$ & \\
\hline \multirow[b]{2}{*}{$\begin{array}{l}\text { Obligation } \\
\text { d'assurance }\end{array}$} & $\begin{array}{l}\text { Obligation individuelle de bénéficier d'une assurance santé (publique, } \\
\text { privée, collective ou individuelle) répondant à des standards minima } \\
\text { de qualité, hors exemptions ou de s'acquitter d'une pénalité financière. }\end{array}$ \\
\hline & $\begin{array}{l}\text { Entreprises de plus de } 50 \text { salariés : obligation de proposer une couver- } \\
\text { ture répondant à des standards de qualité et dont le reste à charge } \\
\text { pour le salarié ne doit pas dépasser } 9,8 \% \text { de ses revenus annuels } \\
\text { et couvrir au minimum } 60 \% \text { des frais médicaux encourus. }\end{array}$ \\
\hline \multirow{3}{*}{$\begin{array}{l}\text { Mesures } \\
\text { financières } \\
\text { et incitatives }\end{array}$} & $\begin{array}{l}\text { Individus : subventions fondées sur les revenus et dégressives à desti- } \\
\text { nation des individus jusqu'à } 400 \% \text { du seuil de pauvreté (soit } 52000 \$ \text {, } \\
\text { ou } 42933 \text { euros, pour un individu et } 80000 \$ \text {, ou } 66051 \text { euros, pour } \\
\text { une famille avec deux enfants. }\end{array}$ \\
\hline & $\begin{array}{l}\text { PME de moins de } 25 \text { salariés et travailleurs indépendants : réductions } \\
\text { fiscales couvrant jusqu'à } 50 \% \text { du coût de l'assurance santé. }\end{array}$ \\
\hline & $\begin{array}{l}\text { Expansion de Medicaid à l'ensemble des individus jusqu'à } 138 \% \\
\text { du seuil de pauvreté fédéral dans les États participant au dispositif. }\end{array}$ \\
\hline \multirow{7}{*}{$\begin{array}{l}\text { Mesures } \\
\text { réglementaires }\end{array}$} & $\begin{array}{l}\text { Création de centrales d'achats pour aider les individus à se procurer une } \\
\text { couverture santé et inciter les PME à en proposer une à leurs salariés. }\end{array}$ \\
\hline & $\begin{array}{l}\text { Création d'un panier de soins essentiels incluant hospitalisation et visite } \\
\text { médicale, soins préventifs, grossesse et maternité, santé mentale, médi- } \\
\text { caments prescrits, soins liés à la toxicomanie, analyses en laboratoire. }\end{array}$ \\
\hline & Instauration d'une limite sur les frais administratifs perçus par les assureurs. \\
\hline & $\begin{array}{l}\text { Interdiction de limiter le montant des dépenses de soins (annuellement } \\
\text { ou à vie) et la durée de la couverture santé. }\end{array}$ \\
\hline & $\begin{array}{l}\text { Interdiction de refuser une couverture santé à des personnes avec des } \\
\text { antécédents médicaux et limitation de la tarification au risque. }\end{array}$ \\
\hline & $\begin{array}{l}\text { Instauration de limites à l'augmentation annuelle du prix des polices } \\
\text { d'assurance. }\end{array}$ \\
\hline & $\begin{array}{l}\text { Possibilité pour les parents de couvrir leurs enfants jusqu'à l'âge de } \\
26 \text { ans. }\end{array}$ \\
\hline
\end{tabular}

* Pour une synthèse plus détaillée de l'ensemble des dispositions de l'Obamacare, voir notamment : https:// www.kff.org/health-reform/fact-sheet/summary-of-the-affordable-care-act/.

régulation des assurances évoqués précédemment (Frean et al., 2017). L'expansion de Medicaid a permis à plus de 15 millions de personnes de bénéficier d'une assurance maladie. Le programme couvre aujourd'hui plus de $20 \%$ de la population américaine, soit environ 65 millions de personnes ${ }^{12}$. Fin 2020, 39 États fédérés sur 50 ont adopté l'expansion de Medicaid prévue par l'Obamacare ${ }^{13}$. Ces adhésions conservent un caractère partisan, les États ayant refusé cette mesure sont connus comme des bastions républicains, à l'image du Texas, de l'Oklahoma, du Kansas, de l'Alabama,

12. Voir Congressional Resarch Service (2021) ; « Status of State Medicaid Expansion Decision: Interactive Map », KFF, April 13, 2021, http://bit.ly/2OBD4Kn. Ce nombre est porté à 72 millions si l'on ajoute le programme CHIP (Children Health Insurance Program) qui est lié à Medicaid.

13. http://bit.ly/3uag99e. 
du Tennessee, du Mississipi et encore de la Floride. Dans ces États conservateurs qui pratiquent des politiques sociales restrictives, Medicaid conserve un caractère ciblé où les seuils et conditions d'éligibilité sont très stricts et des inégalités d'accès aux soins importantes subsistent ${ }^{14}$. Si l'ensemble des États avait étendu Medicaid, comme prévu originairement par l'Obamacare ${ }^{15}$, le think-tank Kaiser Family Foundation estime que le nombre de personnes non assurées aurait baissé de 5 millions supplémentaires (Garfield et al., 2020).

À côté de Medicaid, le dispositif des Health Insurance Marketplaces permet à plus de 11 millions de personnes de bénéficier en 2019 d'une assurance santé individuelle subventionnée et régulée. Ce type d'assurance couvre aujourd'hui $6 \%$ de la population américaine (contre $5 \%$ avant la réforme) ${ }^{16}$. Cinq millions de personnes auparavant non assurées souscrivent une assurance via les marketplaces, et le reste change d'assurance au profit de ce dispositif (Glied et al., 2020). Contrairement à ce qui a été craint en 2010, la croissance des assurances individuelles grâce à l'Obamacare ne se fait pas au détriment des assurances professionnelles. Après une baisse de ces dernières après la récession de 2008, les assurances professionnelles restent la source principale d'assurance santé et elles recommencent au contraire à augmenter à partir de 2014 - moment où les Health Insurance Marketplaces et l'obligation pour les employeurs de plus de 50 salariés de fournir à leurs salariés une assurance santé sont mis en œuvre - pour se stabiliser à leur niveau de la fin des années 1990, soit 156 millions de personnes (Rae et al., 2020).

Les assurances individuelles progressent grâce à l'Obamacare, mais sans doute moins que ne l'ont espéré les architectes de la réforme de 2010, et les dispositifs de stabilisation du marché mis en place ne permettent pas d'en corriger toutes les défaillances. Ces limites sont en partie financières, liées aux contraintes budgétaires et politiques qui ont entouré le vote de la réforme en 2010, dont le coût total devait être limité à 900 milliards de dollars sur dix ans. Une des conséquences de ce budget contraint a été de limiter les subventions aux personnes gagnant moins de $400 \%$ du seuil de pauvreté fédéral, donc de créer des effets de palier pénalisant les classes moyennes. Si les subventions ont relativement bien marché pour les classes populaires et personnes à faibles revenus, dont environ 10 millions reçoivent des subventions liées à l'Obamacare, le média spécialisé Kaiser

14. Dans ces États, les individus à la fois non éligibles à Medicaid et dont les revenus se situent en deçà de $138 \%$ du seuil de pauvreté fédéral - montant à partir duquel les subventions de l'Obamacare démarrent pour souscrire une assurance dans les Health Insurance Marketplaces - souffrent d'un effet de palier important car ils ne bénéficient d'aucune aide publique.

15. Cette disposition est bloquée par la Cour suprême en 2012 (NFIB vs. Sebelius), estimant qu'elle violait les compétences des États fédérés. Suite à ce jugement, l'expansion de Medicaid est devenue facultative.

16. http://bit.ly/3qzdbsQ. 
Health News estime qu'environ 5 millions de personnes de classe moyenne ne reçoivent aucune aide et font face à des coûts d'assurance très élevés ${ }^{17}$. De façon liée, des phénomènes d'« anti-sélection » ${ }^{18}$ sont observés de la même manière avant et après l'ACA (Glied, Jackson, 2018).

Plus profondément, comme nous le montrons plus bas, le secteur des assurances, tout particulièrement le segment des assurances individuelles et des assurances pour PME et travailleurs indépendants peine encore à fonctionner indépendamment d'une action de soutien - et de subvention massive - des pouvoirs publics.

\section{Les difficultés initiales des Health Insurance Marketplaces}

Les Health Insurance Marketplaces, qui sont l'une des mesures phares de l'Obamacare, entrent en fonction en janvier 2014. À mi-chemin entre comparateurs d'assurances et agences de régulation, ces structures facilitent la souscription d'une assurance santé. Statutairement, elles peuvent être gérées soit par les États qui l'ont choisi volontairement, soit par l'État fédéral pour ceux qui ont refusé d'instaurer et de gérer ces organisations. En général, ce choix est guidé par des raisons politiques. Les États démocrates, soutenant l'Obamacare, ont davantage choisi de créer leur propre marketplace (aujourd'hui une minorité de 19 États), tandis que les États républicains hostiles à la réforme (une trentaine) ont plutôt laissé le gouvernement fédéral créer et gérer leur marketplace locale. Conjuguées aux obligations d'assurance et aux subventions et réductions fiscales, les marketplaces doivent améliorer la compétition entre assureurs, faciliter le choix des consommateurs et limiter la croissance des dépenses de santé. Pour autant, les premières années de ces structures sont difficiles, caractérisées par une forte incertitude des assureurs qui les ont conduits à sous-évaluer les besoins des nouveaux participants au dispositif de l'Obamacare, une population majoritairement à faibles revenus, longtemps privée de protection maladie ou mal assurée, dont les besoins en soins médicaux excèdent toutes les prévisions (Holahan et al., 2016). Ce phénomène est moins marqué dans les États qui ont étendu Medicaid, comme la Californie et le Michigan, où Medicaid absorbe une partie de cette population mais est néanmoins présent (Morrisey et al., 2017).

Les polices proposées au sein des Health Insurance Marketplaces sont d'abord relativement bon marché. Elles atteignent en moyenne 270 dollars par mois, soit 224 euros, en 2014 et 2015 pour les assurances de base

17. http://bit.ly/3atfbgP.

18. En économie de l'assurance, le terme d'anti-sélection désigne une situation d'asymétrie d'information dans laquelle seuls les individus ayant besoin de soins médicaux contractent une assurance santé, menant les compagnies d'assurance à augmenter leurs tarifs pour absorber les surcoûts engendrés. 
(les plans « silver $")^{19}$. Mais les assureurs subissent des pertes financières importantes (Cox et al., 2017), ce qui entraîne un réajustement des prix des polices et une forte augmentation des tarifs dans les années qui suivent, autour de $20 \%$ pour la seule année 2017, les plans de base passant en moyenne à 356 dollars par mois ( 295 euros) en 2017 et 481 dollars (398 euros) en $2018^{20}$.

Outre l'augmentation des tarifs, les assureurs participant aux marketplaces réagissent également aux pertes des premières années en proposant des polices de moins bonne qualité, limitant le nombre et la variété des professionnels de santé accessibles aux nouveaux assurés et augmentant les frais restant à charge des assurés et les franchises. Ce phénomène touche surtout les assurances de base destinées aux classes moyennes et cible principale des subventions prévues par l'Obamacare. Au départ, un nombre substantiel de contrats proposent un accès aux établissements et professionnels de santé relativement ouvert dans lesquels il est possible de consulter des professionnels n'appartenant pas au réseau de base de l'assureur moyennant un surcoût modéré (Preferred Providers Organizations), mais ces polices se sont aujourd'hui raréfiées et la norme est un réseau local peu étendu et fermé, de type Health Maintenance Organizations (HMO), moins cher car permettant à l'assureur de négocier des tarifs préférentiels avec le professionnel de santé en échange de l'assurance d'un volume de patients. Ces recompositions des contrats augmentent le phénomène de la «sous-assurance » ou de l'assurance inadéquate, qui, selon un rapport récent du Commonwealth Fund, touche encore aujourd'hui et en dépit des garanties votées par l'Obamacare plus de $40 \%$ des personnes ayant souscrit une assurance individuelle (Collins et al., 2019).

Ensuite, un tri important se fait parmi les compagnies d'assurance au cours de ces premières années, avec certains assureurs comme le géant United Health qui déclarent tout simplement en avril 2016 ne plus vouloir participer à l'Obamacare car perdant trop d'argent ${ }^{21}$. De fait, le nombre et surtout la variété des assureurs présents au sein des marketplaces diminue après trois ans. Les assureurs qui s'en sortent le mieux sont des HMO « low-cost » spécialisés dans les plans Medicaid, et qui, profitant déjà d’un réseau de professionnels de santé à des tarifs compétitifs, peuvent proposer des assurances à bas prix ${ }^{22}$. Les assureurs également très implantés locale-

19. L'ACA crée quatre catégories de plans d'assurance santé : les plans "bronze », " silver », " gold » et " platinium ». Ils diffèrent en fonction de la générosité des prestations, du montant de la police mensuelle, du montant des franchises et de la qualité du réseau de médecins et d'hôpitaux auxquels les assurés ont accès. Les plans silver constituent le standard fédéral, qui ouvrent la possibilité de recevoir des aides financières du gouvernement fédéral (premiums subsidies et cost sharing reductions).

20. http://bit.ly/3s00WWo.

21. C. Y. Johnson, "UnitedHealth Group to exit Obamacare exchanges in all but a "handful" of states », The Washington Post, April 19, 2016, http://wapo.st/3udsEAX.

22. C'est le cas par exemple de Molina Healthcare qui est un assureur national spécialisé dans les produits Medicaid et qui domine le marché en Californie, dans le Michigan, au Texas et en Floride. 
ment comme Blue Cross Blue Shield résistent bien aussi, tout comme des assureurs régionaux ayant développé des partenariats privilégiés avec des systèmes de santé locaux (systèmes hospitaliers et médecine de ville) ${ }^{23}$. En revanche, certains types d'assureurs ne survivent pas aux premières années de l'Obamacare. C'est le cas des Co-ops, les coopératives d'assureurs à but non lucratif fonctionnant un peu à la manière de nos mutuelles. Ces Co-ops sont soutenues en 2010 par l'aile gauche du Parti démocrate. En théorie, ces structures auraient dû proposer des tarifs plus abordables que les assurances à but lucratif et par effet de compétition tirer les tarifs des polices vers le bas. Mais dans les faits, elles ne sont tout simplement pas rentables. Les Co-ops proposent entre 2014 et 2016 les tarifs les moins élevés parmi les assureurs participant aux marketplaces, mais elles se révèlent non viables économiquement et un grand nombre d'entre elles font faillite.

Enfin, des différences géographiques importantes apparaissent selon les États, qui exacerbent ces difficultés dans certaines régions. Le marché des assurances individuelles est devenu plus fragmenté et très hétérogène selon les États et au sein même des États d'un comté à l'autre en fonction des caractéristiques des territoires et du tissu médical local (Morissey et al., 2017). Le prix des polices pratiquées dans les différentes Health Insurance Marketplaces présente des variations du simple au triple selon les États. Ainsi, en 2020, les polices de base (silver) atteignent un peu plus de 880 dollars (729 euros) par mois dans le Wyoming, mais seulement 309 euros (256 euros) dans le Minnesota ${ }^{24}$. Le nombre d'assureurs en compétition au sein des marketplaces est également très variable. Alors qu'il s'élève en 2020 en moyenne à 4,5 compagnies d'assurance, dans plusieurs États comme le Wyoming, le Delaware, l'Alaska et encore l'Arizona, il n'existe aucune compétition et un seul assureur est présent. Dans l'État de l'Iowa, l'absence d'attractivité de la marketplace est telle qu'en 2018, aucun assureur ne veut y participer et l'État est contraint de mettre en place un système de réassurance - subventionnant fortement les assureurs en cas de frais médicaux élevés pour les faire revenir (Parys, 2018). À l'inverse, certains États sont plus richement dotés et une réelle compétition existe : le comté de Los Angeles compte sept assureurs, ou encore les comtés autour de New York City en comptent six ${ }^{25}$. Ces disparités mettent en lumière la complexité des mesures de régulation du marché des assurances individuelles aux États-Unis, car il ne s'agit pas d'encadrer un marché unique, mais plutôt une multitude de marchés avant tout locaux, dont les spécificités dépendent des caractéristiques du territoire. En règle générale, l'Obamacare fonctionne mieux dans des zones urbaines et aisées, richement dotées en

23. À l'image d'Optima Health Plans qui est une émanation du système hospitalier Sentara en Virginie, qui regroupe plus de 300 centres de santé en ambulatoire, une douzaine d'hôpitaux, autant de maisons de repos (nursing centers). II s'agit du troisième plus grand employeur de Virginie.

24. http://bit.ly/2M1805R

25. http://bit.ly/2ZsMEBv. 
prestataires de santé. Dans les zones plus rurales et plus pauvres, dans des déserts médicaux, la compétition entre plans d'assurance ne fonctionne pas, les tarifs pratiqués par les assureurs sont plus élevés. Il faut ajouter que les Health Insurance Marketplaces gérées par le gouvernement fédéral au sein d'États hostiles à l'ACA ayant refusé de participer au dispositif, ont probablement connu des difficultés initiales plus importantes (Jones, Greer, 2013).

Ceci étant dit, après des mesures d'ajustement assez brutales entre 2016 et 2018, les Health Insurance Marketplaces semblent se stabiliser au niveau fédéral. Les assureurs déclarent des marges positives en 2019 et 2020 et un nombre croissant de compagnies d'assurance montre une volonté de réintégrer le dispositif. Les tarifs moyens des polices baissent aussi légèrement entre 2018 et 2020, passant de 481 dollars (398 euros) en moyenne à 462 dollars (382 euros) ${ }^{26}$ pour les plans de base, en dépit des mesures prises par l'administration Trump et en particulier la suppression de la pénalité liée à l'obligation individuelle d'assurance (voir infra). L'incertitude sur l'avenir de la réforme reste forte toutefois, car la constitutionnalité de l'Obamacare est menacée par une action en justice menée en 2020 par 18 États républicains devant la Cour suprême au motif que la loi est inconstitutionnelle depuis l'abrogation de la pénalité liée au mandat individuel en 2017 (voir infra). La nomination de la juge conservatrice Amy Coney Barrett à l'extrême fin du mandat de Donald Trump à la place du siège de Ruth Bader Ginsburgh renforce ce risque et fait peser une épée de Damoclès sur la réforme.

Notons enfin que ces observations s'appliquent également aux SHOP Marketplaces pour les petites et moyennes entreprises bien que les données soient assez peu nombreuses en comparaison aux données liées aux marketplaces opérant sur le segment des assurances individuelles. Gabel et alii (2015) montrent que ces structures offrent en général moins de choix que les assurances existant en dehors et moins régulées. Certains États ruraux comme l'Alabama, le Maine, le Kansas et encore le Tennessee ne comptent que deux assureurs présents dans ces marketplaces, alors que d'autres États comme le Maryland, le Michigan, New York et la Pennsylvanie comptent entre 9 et 10 assureurs proposant des assurances régulées et ouvrant droit à des aides financières dans leurs échanges. Ces plans sont cependant en général entre 4 et $5 \%$ moins chers que les polices équivalentes proposées en dehors des marketplaces.

\section{L'obligation individuelle d'assurance en question}

Autre mesure majeure de l'Obamacare, l'obligation individuelle d'assurance cristallise les débats politiques récents entre Démocrates et Républicains, éclipsant les controverses autour de l'obligation d'assurance des

26. http://bit.ly/2ZvLOPw. 
entreprises, qui a pourtant été une des origines de l'échec de la réforme Clinton dans les années 1990. L'obligation individuelle d'assurance et la pénalité financière l'accompagnant, dont doivent s'acquitter ceux qui ne souscrivent pas d'assurance santé, est immédiatement impopulaire et critiquée par les Républicains dès l'origine, alors que son efficacité et son équité sont aujourd'hui en question (Gais, Gusmano, 2020). En 2010, elle a été négociée avec les compagnies d'assurance pour limiter les phénomènes d'anti-sélection qui portent les seuls individus malades à souscrire une assurance santé. En échange de cette garantie de voir davantage de personnes en bonne santé souscrire un contrat, les assureurs ont accepté les régulations créées par l'Obamacare : notamment les garanties minimales de couverture et l'abandon de la tarification au risque. Cette mesure est alors apparue relativement consensuelle parmi les Démocrates. Elle s'appuie sur des expériences menées dans les années 2000 au niveau des États fédérés et en particulier sur la réforme du Massachussetts en 2006 qui a permis de faire massivement baisser le taux de non-assurés (Beaussier, 2016:165). Pour autant, cette mesure ne se révèle pas suffisante pour stabiliser les Health Insurance Marketplaces, elle n'empêche pas les phénomènes d'antisélection, de free riding et les augmentations importantes du prix des polices participant aux marketplaces.

Si pour les Démocrates cette mesure représente un pilier de l'Obamacare, elle constitue une mesure liberticide et injuste pour le camp républicain, qui n'a de cesse à partir de 2012 d'obtenir son abrogation. Celle-ci est obtenue de fait lors de l'adoption du Tax Cut and Job Act en 2017, la grande réforme fiscale des Républicains, qui supprime l'amende qu'encourt ceux qui ne respectent pas l'obligation d'assurance ${ }^{27}$. Cela revient dans les faits à annuler cette mesure et c'est l'action la plus significative que les Républicains ont menée sur l'Obamacare. Elle fait craindre à beaucoup d'observateurs des politiques de santé américaines que l'ensemble de l'architecture de l'Obamacare ne s'effondre, tout particulièrement le dispositif des marketplaces et avec lui les protections des consommateurs mises en œuvre par Obama. Cependant, un tel scénario ne s'est pas encore réalisé. Le nombre de personnes non assurées a depuis recommencé à augmenter mais pas autant que les estimations d'alors ne l'envisageaient. 1,2 million de personnes ont perdu leur assurance santé (Tolbert et al., 2020), loin des 4 millions de personnes anticipées par le Congressional Budget Office (CBO, 2017) ${ }^{28}$. Surtout, il n'est pas établi que ce soit la suppression de cette mesure, par comparaison par exemple à la hausse des tarifs des assurances proposées

27. Cette pénalité pouvait atteindre 700 dollars, soit 580 euros, par adulte et 350 dollars (290 euros) par enfant d'un foyer. Ce qui signifie qu'une famille de quatre personnes encourait jusqu'à 2100 dollars, soit 1739 euros d'amende pour non-respect de l'obligation individuelle d'assurance.

28. Le Congressional Budget Office table en novembre 2017 sur une augmentation de 4 millions de personnes non assurées en 2019, une augmentation qui doit atteindre 13 millions en 2027. 
dans les Health Insurance Marketplaces, qui explique cette augmentation. Des études relativisent aujourd'hui l'effet de l'obligation d'assurance sur les gains de couverture et montrent que l'expansion de Medicaid et les subventions versées pour participer au dispositif des marketplaces expliquent les gains de couverture, alors que la pénalité et l'effet incitatif de l'obligation individuelle sont quasi-nuls (Frean et al., 2017). Fréquemment, les personnes restant non assurées sont des personnes à faibles revenus qui n'ont simplement pas les moyens de souscrire une assurance santé. Non éligibles à Medicaid - ou résidant dans un État qui n'a pas étendu Medicaid - en dépit des subventions prévues par l'Obamacare, ces groupes de population n'ont pas les moyens de se payer une assurance santé (Tolbert et al., 2019). De façon liée, plusieurs voix s'élèvent aujourd'hui à gauche du spectre politique pour dénoncer l'iniquité de cette pénalité accompagnant l'obligation individuelle d'assurance et la nature régressive de cette taxe pesant en majorité sur les personnes à revenus faibles ou moyens (Vermont Individual Mandate Working Group, 2018).

Avec la suppression de la pénalité au niveau fédéral, la question est transférée aux États fédérés, qui doivent décider d'adopter ou non une mesure similaire à leur échelle territoriale. De ce point de vue, les positions adoptées sont assez hétérogènes (Gais, Gusmano, 2020). Une fracture partisane existe : les États conservateurs comme le Texas ou la Floride, fermement opposés à l'Obamacare, suppriment simplement le dispositif alors qu'ils envisagent des mécanismes alternatifs de stabilisation du marché comme des programmes de réassurance ou des subventions aux assurances ciblées sur des groupes de personnes atteintes de maladies chroniques (high risk pools), revenant à établir un transfert de risque des assureurs vers les pouvoirs publics. Les États démocrates soutenant l'ACA mènent une réflexion de fond sur la manière de protéger les acquis de l'Obamacare en l'absence de pénalité financière. Certains réintroduisent cette obligation à l'identique ${ }^{29}$ sur leur territoire comme Washington DC, d'autres modifient le dispositif comme le New Jersey, le Rhode Island, la Californie ou encore le Maryland.

Le fait que la pénalité financière soit dorénavant une responsabilité des États octroie à ces derniers une marge de manœuvre plus importante sur les fonds récoltés, ce qui permet la mise en place d'expérimentations et d'innovations. Le New Jersey crée ainsi un système de transfert de risques allouant les montants issus de la pénalité à un fonds de réassurance destiné à stabiliser le marché en partageant avec les assureurs les frais engendrés par les patients les plus malades. Le Maryland, le Rhode Island et la

29. En décembre 2019, 9 États ont introduit une obligation individuelle d'assurance dans leur législation : la Californie, Washington DC, le Massachusetts, le New Jersey, le Rhode Island et le Vermont. 5 États supplémentaires sont en voie de le faire : le Connecticut, Hawaï, le Maryland, le Minnesota et l'État de Washington. 
Californie utilisent ces montants pour accroître les subventions fédérales et éliminer les frais restant à charge des assurés (franchises). Le Maryland utilise les revenus de cette pénalité pour augmenter les subventions à destination des foyers à revenus faibles à moyens. Le Connecticut bloque les fonds sur des comptes épargnes santé (personnal health savings accouts) que les intéressés peuvent utiliser pour souscrire une assurance. Washington DC utilise plus simplement les fonds pour mener des campagnes d'information et d'accompagnement du public, un poste de dépense qui est très fortement réduit au niveau fédéral par l'administration Trump.

D'autres États encore qui se situent pourtant à gauche du spectre politique décident simplement de se passer de cette pénalité. Ainsi le Vermont conserve l'obligation d'assurance mais en la vidant de toute dimension punitive car la considérant comme une taxe régressive et inéquitable (Vermont Individual Mandate Working Group, 2018). Les habitants du Vermont doivent mentionner dans leur déclaration d'impôt qu'ils n'ont pas d'assurance et ne répondent pas à l'obligation d'assurance, mais l'administration locale de la santé utilisera ces déclarations pour contacter individuellement chaque non-assuré et lui proposer un accompagnement individualisé. L'État de New York décide simplement que ce dispositif n'est pas utile, et qu'une expansion de Medicaid couplée avec des subventions plus importantes complétant les fonds fédéraux de l'Obamacare atteindraient un meilleur résultat et permettraient à davantage de foyers à faibles et moyens revenus de bénéficier d'une assurance santé. Ainsi New York se positionne avec une proposition alternative, le "Basic Health Plan ", proposant une couverture maladie certes limitée mais bon marché aux personnes non assurées à faibles revenus mais non éligibles à Medicaid (polices mensuelles de l'ordre de 20 dollars) ${ }^{30}$.

\section{L'Obamacare et l'administration Trump : une stratégie de démantèlement progressif}

À côté de l'obligation d'assurance, les Républicains tentent depuis 2011 de démanteler la plupart des mesures réglementaires de l'Obamacare, accusées d'être à l'origine d'une augmentation incontrôlée du prix des polices. Les premières actions menées sous Trump visent à réduire les ressources de l'Obamacare et de ses principaux instruments de financement. Cela commence par des mesures de prévention et de santé publique et par des programmes de recherche sur l'efficacité des procédures médicales. C'est également le cas du fonds de mise en œuvre de l'ACA, qui voit ses ressources plusieurs fois réduites, ce qui augmente les difficultés du Département de la santé et cause des avaries techniques lors de la mise en œuvre des Health Insurance Marketplaces.

30. http://on.ny.gov/3pvNHvf. 
Les Républicains qui ne croient pas à l'architecture de la réforme proposent d'autres mécanismes pour faire baisser le prix des polices d'assurance, appuyés sur des instruments relativement classiques du répertoire d'actions conservateur, qui reviennent à déréguler le marché des assurances et à subventionner massivement les assurances pour qu'elles prennent en charge les patients dont l'état de santé représente un risque financier élevé pour elles. Les dérégulations sont soutenues par l'administration Trump dès 2016. En autorisant les assureurs à proposer des polices de moins bonne qualité et à pratiquer la sélection des risques, il s'agit de leur permettre de commercialiser des polices moins chères pour les personnes en bonne santé, délaissant par là même le principe de solidarité au profit d'une dynamique d'individualisation de la couverture santé.

Une partie de ces mesures de dérégulation passe par des usages répétés des pouvoirs d'exécution de la Maison Blanche. Donald Trump signe ainsi en octobre $2017^{31}$ un décret intitulé « Promoting Healthcare Choice and Competition Across the United States », encourageant des mécanismes de contournement des protections et régulations de l'Obamacare, et permettant aux assureurs de proposer des polices de moindre qualité, mais aussi bien moins chères que les polices proposées au sein des marketplaces. Le décret renforce ainsi le dispositif des "Association Health Plans » autorisant les petites et moyennes entreprises à se regrouper pour proposer à leurs salariés des formules d'assurance non soumises aux régulations et aux garanties de couverture de l'Obamacare et qui autorisent les assureurs à pratiquer une tarification au risque. Le même décret étend également l'accès aux assurances de court terme (dont la durée maximale passe de 3 mois à un an renouvelable trois fois) qui ne sont pas non plus soumises aux protections mises en place par l'Obamacare (Jones et al., 2018).

À côté de ces dérégulations, l'administration Trump soutient également des mécanismes de réassurance ciblés pour faciliter l'assurance des individus les plus malades et in fine remplacer les mécanismes réglementaires par des mécanismes d'incitation financière. Ces instruments sont mis en œuvre principalement au niveau des États fédérés en utilisant une disposition de l'Obamacare qui offre aux États la possibilité d'expérimenter des formes alternatives de couverture, tant que ces expérimentations respectent l'esprit et les objectifs de l'Obamacare. La section 1332 de la loi autorisant ces expérimentations est à l'origine une disposition permettant aux États qui le souhaitent d'aller plus loin que l'Obamacare et d'encourager des innovations politiques notamment en matière de couverture maladie universelle ${ }^{32}$. Cette disposition initialement plutôt marginale de

31. https://bit.ly/3k1MjiP.

32. Le Vermont a par exemple voté un projet de création d'une assurance maladie universelle publique en 2014 en s'appuyant sur la section 1332 de l'ACA. 
la réforme devient importante sous l'administration Trump (Wright et al., 2019), qui encourage en particulier des expérimentations allant dans le sens de la mise en œuvre de fonds de réassurance au niveau des États ou encore de la création de bigh risk-pools (groupes d'assurés à haut risques). Ce transfert de responsabilité aux États fédérés et l'affaiblissement du niveau fédéral constitue un autre axe traditionnel du programme républicain, dont l'administration Trump s'est faite l'écho en encourageant l'autonomisation des États dans la mise en œuvre de l'Obamacare et leur prise de distance vis-à-vis du cadre fédéral. Toutefois toutes les initiatives n'aboutissent pas.

Treize États candidatent pour lancer une expérimentation section 1332, mais seulement quatre d'entre eux sont approuvés. L'arrivée à la Maison Blanche de Trump met un coup d'arrêt aux projets d'expérimentation plus ambitieux, comme le projet de la Californie d'inclure les immigrants en situation irrégulière dans le dispositif de l'Obamacare. Parmi les États dont l'expérimentation est retenue, l'Alaska, le Minnesota et l'Oregon proposent de mettre en place un programme de réassurance pour stabiliser le marché des assurances individuelles en compensant les pertes encourues par les assureurs opérant au sein des Health Insurance Marketplaces. En Alaska, par exemple, le programme rembourse les assureurs pour les frais des assurés ayant une maladie figurant parmi une liste de 33 maladies à coût élevé. L'Oregon rembourse les assureurs une fois qu'un assuré atteint un certain plafond de dépenses. D'autres États comme le Maine, le Wisconsin, le Maryland, l'Idaho, le Kentucky, la Louisiane, le New Hampshire, le New Jersey, le Rhode Island, et le Texas déposent ou sont en voie de déposer une candidature pour lancer une expérimentation pour la création d'un programme de réassurance sans que leur projet n'ait encore abouti. Ces mécanismes se sont révélés jusqu'à présent relativement efficaces, la croissance des prix des polices d'assurance au sein des marketplaces a ralenti et ces structures ont retrouvé une certaine attractivité auprès des assureurs (Wright et al., 2019). Toutefois, on voit mal en quoi le fait de subventionner massivement les compagnies d'assurance pour qu'elles proposent des polices moins chères aux individus en bonne santé et qu'elles acceptent de couvrir les personnes malades est réellement une avancée sur le long terme, ni en quoi de telles mesures permettent de stabiliser ce marché durablement hors de ces injections massives d'argent public. Surtout la valeur ajoutée de ces mesures de réassurance à la fois au niveau économique et au niveau éthique n'est pas claire en comparaison par exemple des propositions démocrates de créer un programme public ouvert à tous sur une base volontaire. 


\section{Conclusion : réformer la réforme, la santé dans le programme électoral de Joe Biden}

L'Obamacare est considérée comme la réforme la plus significative des politiques de santé américaines depuis la création de Medicare et Medicaid en 1965. Dix ans après son vote par le Congrès, elle a réalisé de réelles avancées en termes de couverture de la population et demeure relativement indemne malgré les conflits politiques qu'elle a suscités entre Républicains et Démocrates. Il n'était pas dans l'objectif de cet article de faire le tour des avancées réalisées par cette réforme, mais plus simplement, de comprendre l'impact qu'elle a eu sur le marché des assurances santé. Ce bilan peut être résumé en quelques points.

Tout d'abord, il n'y a pas eu un transfert majeur des formes d'assurance préexistantes vers l'assurance individuelle et les marketplaces. Cellesci ont attiré près de 11 millions de personnes, mais cela représente une progression de 5 millions de personnes depuis 2010. Les assurances individuelles couvrent aujourd'hui $6 \%$ de la population contre $5 \%$ en 2010 . Les avancées de couverture que l'on a pu constater ont été principalement le fait d'autres dispositifs de la réforme, à commencer par l'expansion de Medicaid. Les assurances professionnelles sont restées stables et ont même progressé en dix ans, ce qui contredit les prévisions pessimistes selon lesquelles l'Obamacare signait la fin de la couverture collective.

Ensuite, des disparités territoriales importantes ont émergé dans la mise en œuvre de la réforme, et des marketplaces. Ces disparités mettent en lumière la complexité des mesures de régulation des assurances aux ÉtatsUnis, car il ne s'agit pas d'encadrer un marché unique, mais plutôt une multitude de marchés avant tout locaux, dont les spécificités dépendent des caractéristiques du territoire, de la population et des acteurs politiques en charge de la mise en œuvre de la réforme. Comme nous l'avons souligné, l'Obamacare fonctionne mieux dans des zones urbaines et aisées, richement dotées en prestataires de santé - zones en outre fréquemment démocrates. Dans les zones plus rurales et plus pauvres, également plus conservatrices, la compétition entre plans d'assurance ne fonctionne pas, les tarifs pratiqués par les assureurs sont plus élevés. L'ambition de la réforme, qui était de proposer un cadre réglementaire unifié au niveau fédéral, se heurte aux caractéristiques locales, au nombre d'assureurs en compétition, au tissu médical. Ces caractéristiques appellent peut-être à une différenciation de l'action publique selon les territoires. C'est en tout cas la direction qui semble être empruntée aujourd'hui car les États ont de plus en plus de marges de manœuvre dans la mise en œuvre de l'Obamacare.

Le troisième et dernier point renvoie à l'idée que les limites évoquées plus haut portent en germe de futures réformes dont les contours ont été 
discutés dans le contexte de l'élection présidentielle de novembre 2020. Du côté démocrate, la subsistance d'un nombre encore important de personnes sans accès aux soins (autours de 30 millions) tout comme les difficultés récurrentes du marché des assurances santé ont rendu audibles les voix les plus à gauche et apporté une nouvelle crédibilité à des dispositifs d'action publique inenvisageables auparavant comme la création d'une protection maladie publique pour l'ensemble de la population. Il semble exister aujourd'hui un relatif consensus au sein du Parti démocrate autour de la création d'une «option publique » qui étendrait les conditions d'éligibilité aux programmes existants et en particulier Medicare et Medicaid sans pour autant se substituer complètement aux assurances privées. Point de division majeur de la majorité démocrate au Congrès en 2010, le thème de la création d'une public option revient aujourd'hui en force. Elle est désormais soutenue par une majorité de Démocrates et son insistance sur la notion de « choix » entre une assurance privée et publique dans le contexte post-Obamacare pourrait lui valoir le soutien des centristes.

C'est en tous cas le programme soutenu par le nouveau Président Joe Biden, dont les majorités au Sénat et à la Chambre des représentants pourraient lui fournir une fenêtre d'opportunité pour initier une telle réforme de la réforme. Toutefois l'implacable arithmétique du Congrès américain risque bien de l'en empêcher. La majorité démocrate au Sénat en particulier (50 sénateurs sur 100 plus la voix de la Vice-Présidente Kamala Harris) apparait trop ténue pour faire passer une mesure de ce type d'ici les deux prochaines années et notamment surmonter la menace du filibustering que les Républicains ne manqueront pas de brandir face à toute réforme de santé d'ampleur et qui nécessiterait 60 sénateurs sur 100 pour la surmonter.

Côté républicain, bien que le bilan de la présidence de Trump en matière de santé soit mauvais et aggravé par une gestion désastreuse de la pandémie de Covid-19, les années Trump ont permis l'émergence et l'expérimentation de nouveaux mécanismes de soutien du marché des assurances privées qui sortent des outils traditionnels des politiques sociales et empruntent au registre de l'assurance et de l'économie de l'assurance. Le programme santé républicain, tel qu'esquissé depuis quatre ans, semble se diriger vers la promotion de mécanismes de réassurance au travers desquels l'État garantirait et encouragerait la solvabilité des assureurs privés, tout en mettant en œuvre des subventions importantes aux assurances pour qu'elles prennent en charge les personnes ou les maladies particulièrement chères (les high risk. pools). 


\section{Références bibliographiques}

Beaussier A.-L. (2016), La santé aux États-Unis : une histoire politique, Paris, Presses de Sciences Po.

Butler S.M., HaisImaier E.F. (eds.) (1989), A National Health System for America, Washington DC, Heritage Foundation Press.

CBO (2017), Repealing the Individual Health Insurance Mandate: An Updated Estimate, Report, Congressional Budget Office, November, https://www.cbo. gov/publication/53300.

CBO (2019), Federal Subsidies for Health Insurance Coverage for People Under Age 65: 2019 to 2029, Congressional Budget Office, May, https://www.cbo. gov/system/files/2019-05/55085-HealthCoverageSubsidies_0.pdf.

Collins S.R., Bhupal H.K., Doty M.M. (2019), « Health Insurance Coverage Eight Years After the ACA: fewer uninsured Americans and shorter coverage gaps, but more uninsured ", Issue Briefs, The Commonwealth Fund, February 7 , http://bit.ly/3jYZu48.

Congressional Research Service (2021), " US health care coverage and spending », In Focus, CRS, January 26, https://fas.org/sgp/crs/misc/lF10830.pdf.

Cox C., Levitt L., Claxton G. (2017), « Insurer financial performance in the early years of the Affordable Care Act », Data Note, Kaiser Family Foundation, April 21, https://bit.ly/2ZrZ5gR.

Frean M., Gruber J., Sommers B.D. (2017), « Premium subsidies, the mandate, and Medicaid expansion: Coverage effects of the Affordable Care Act ", Journal of Health Economics, ${ }^{\circ}$ 53, p. 72-86, https://doi.org/10.1016/j. jhealeco.2017.02.004.

Gabel J.R., Stromberg S.T., Green M., Lischko A., Whitmore H. (2015), « An early look at SHOP marketplaces: Low premiums, adequate plan choice In many, but not all, States », Health Affairs, vol. 34, n 5, p. 732-740, https://doi. org/10.1377/hlthaff.2014.1370.

Gais T.L., Gusmano M.K. (2020), "Putting the pieces together again: American States and the end of the ACA's shared responsibility payment ", Journal of Health Politics, Policy and Law, vol. 45, $\mathrm{n}^{\circ} 3$, p. 439-454, https://doi. org/10.1215/03616878-8161048.

Garfield R., Orgera K., Damico A. (2020), « The coverage gap: Uninsured poor adults in States that do not expand Medicaid ", Issue Brief, Kaiser Family Foundation, January 14, https://bit.ly/3quZISW.

Glied S.A., Collins S.R., Lin S. (2020), « Did the ACA lower Americans' financial barriers to health care?: A review of evidence to determine whether the Affordable Care Act was effective in lowering cost barriers to health insurance coverage and health care ", Health Affairs, vol. 39, $n^{\circ} 3$, p. 379-386, https:// doi.org/10.1377/hlthaff.2019.01448.

Glied S.A., Jackson A. (2018), « Who entered and exited individual market before and after ACA? ", Issue Brief, The Commonwealth Fund, https://bit. ly/2ZoM87D.

Holahan J., Blumberg L.J., Wengle E. (2016), « What does the failure of some Coops and the possible pullout of United Healthcare mean for the Affordable Care Act? », In-Brief, Urban Institute, January, https://urbn.is/3jZqaBO. 
Howard C. (1997), The Hidden Welfare State: Tax Expenditures and Social Policy in the United States, Princeton, Princeton University Press.

Jones D.K., Greer S.L. (2013), « State politics and the creation of Health Insurance Exchanges ", American Journal of Public Health, vol. 103, $n^{\circ}$ 8, e8-e10, https://doi.org/10.2105/AJPH.2013.301429.

Jones D.K., Gusmano M.K., Nadash P., Miller E.A. (2018), « Undermining the ACA through the executive branch and federalism: What the Trump administration's approach to health reform means for older Americans », Journal of Aging \& Social Policy, vol. 30, $\mathrm{n}^{\circ} 3-4$, p. 282-299, https://doi.org/10.1080/ 08959420.2018.1462684.

Morrisey M.A., Rivlin A.M., Nathan R.P., Hall M.A. (2017), « Five-State study of ACA marketplace competition: A summary report », Risk Management and Insurance Review, vol. 20, $\mathrm{n}^{\circ}$ 2, p. 153-172, https://doi.org/10.1111/rmir.12079.

Parys J.V. (2018), « ACA marketplace premiums grew more rapidly in areas with monopoly insurers than in areas with more competition ", Health Affairs, vol. 37, n 8, p. 1243-1251, https://doi.org/10.1377/hlthaff.2018.0054.

Rae M., McDermott D., Levitt L., Claxton G. (2020), « Long-term trends in employerbased coverage ", Briefs, Kaiser Family Foundation, April 3, https://www.healthsystemtracker.org/brief/long-term-trendsin-employer-based-coverage/.

Tolbert J., Orgera K., Damico A. (2020), « Key facts about the uninsured population », Issue Brief, December 13, http://bit.ly/3psikl5

Vermont Individual Mandate Working Group (2018), Individual Mandate: Report of the Individual Mandate Working Group, November, https://bit.ly/3u45Un0.

Wright B., Porter A., Singer P.M., Jones D.K. (2019), « The devolution of health reform? A comparative analysis of State innovation waiver activity ", Journal of Health Politics, Policy and Law, vol. 44, n 2, p. 315-331, https:// doi.org/10.1215/03616878-7277404. 